\title{
SNR-aware and power-efficient multicast cooperative routing algorithm in wireless networks
}

\author{
Hong-Hsu Yen
}

\begin{abstract}
Power-efficient multicast routing is an active research field in wireless networks because most network nodes are powered by battery. However, this kind of multicast routing only considers the transmission radius coverage without addressing whether the signal quality is able to meet bandwidth requirement of the users. In this article, by leveraging on the Maximum Ratio Combining signal processing technique, a novel cooperative multicast routing scheme that meets the Signal-to-Noise Ratio (SNR) requirements of the traffic demands in power efficient way is developed. This problem is formulated as an optimization problem where the objective is to minimize the total transmission power subject to the SNR constraint. This is a challenging cross-layer design problem to simultaneously consider the cooperative routing in the network layer and the SNR-aware power control in the physical layer. A heuristic algorithm called bandwidth-aware cooperative radius adjustment (BACRA) is proposed to tackle this problem. The basic idea of BACRA is to select the node with the maximum ratio of contributed SNR to the power (denoted as SNR/P) to expand its power one at a time until the SNR requirements are all satisfied. The BACRA is proved to be optimal in terms of the SNR/P performance ratio. Numerical results demonstrate that the BACRA outperforms the other heuristics under all tested cases, especially in stringent SNR requirements and sparse network.
\end{abstract}

Keywords: Cooperative routing, SNR QoS, Power control, Minimum power broadcast multicast, Wireless multicast advantage

\section{Introduction}

In wireless network, node power radiation management is an important issue since most of the network nodes are powered by the battery. One interesting property that only exists in wireless networks but not in wired networks is the Wireless Multicast Advantage (WMA) [1]. For WMA, neighbor nodes that are within the range of a sender's transmission radius can receive the transmitted data. This WMA property can be used to reduce the total power consumption for multicast and broadcast applications. Figure 1 depicts an example of the WMA. In this example, node $A$ is the sender node. As the transmission power is large enough to reach, nodes $E, B, C$, and $D$ are also covered. Based on WMA, in Figure 1, the total power to send the data to all the destination nodes

Correspondence: honghsuyen@gmail.com

Department of Information Management, Shih-Hsin University, Taipei, Taiwan is $\Pi_{4}$ instead of $\left(\Pi_{1}+\Pi_{2}+\Pi_{3}+\Pi_{4}\right)$ in wired line communication. Note that the transmission power $\Pi$ is defined in Section "Cooperative routing model for MPBBA".

Algorithms leverage on the WMA property to minimize the total power consumption is an active research in wireless networks. This kind of minimum total power in broadcasting/multicasting routing problem (denoted as the MPB problem) in wireless networks has been shown to be an NP-hard problem [2] and heuristics [1-5] has been proposed to get the near-optimal solutions. However, the MPB problem only considers the transmission radius coverage. It does not address the cases where signal quality may fail to meet the bandwidth demands of the origin-destination (OD) pairs.

The available bandwidth in the wireless link depends on the signal quality and the modulation scheme. If the received signal quality is below the minimum signal 


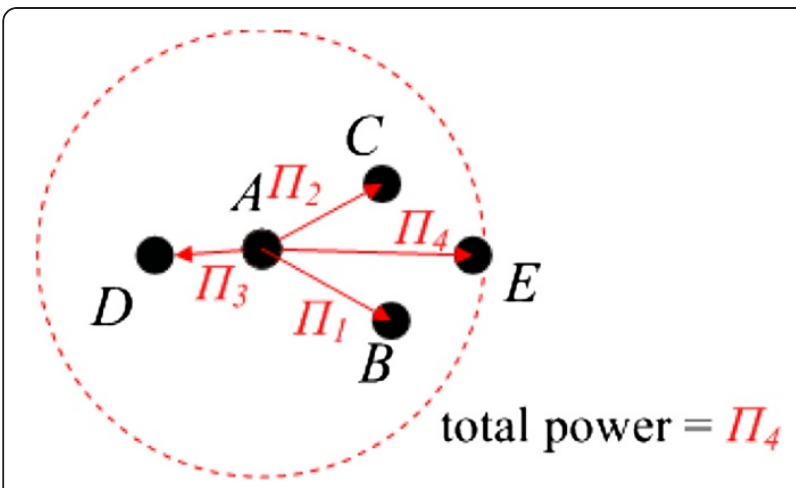

Figure 1 Power savings by WMA.

quality threshold of the modulation scheme, then the receiver could not successfully decode the received information. Therefore, less sophisticated modulation scheme that requires lower signal quality should be adopted to decode the received information. Then, the available bandwidth on this wireless link could be reduced. For example, in IEEE 802.11b, the effective bandwidth could be 1, 2, 5.5, and 11 Mbps [6], which depends on the signal quality of the receiver. In general, when the transmission power at the transmitter is fixed, the shorter the distance between the transmitter and the receiver, the better the signal quality there would be, and more sophisticated modulating scheme (e.g., 64-QAM) could be used to achieve higher data throughput. On the other hand, in the case of long distance between transmitter and receiver, the signal quality is poor so that only ordinary modulating scheme (e.g., QPSK) could be used. Then, the effective bandwidth might fail to meet the traffic demands of the OD pair. This kind of MPB with bandwidth-aware problem (denoted as MPBBA) is different from traditional MPB problem. With considering the bandwidth requirement and power consumption simultaneously, MPBBA problem is more challenging than the MPB problem.

One possible way to tackle the MPBBA problem is first to have the MPB solutions without addressing the bandwidth constraint. Next expanding the transmission radius so that bandwidth requirement could be satisfied. This kind of two-stage algorithm could satisfy the bandwidth requirement but it is not as power efficient as reported in [7]. In [7], we proposed optimization-based heuristic to tackle the MPBBA problem. Even though this optimization-based heuristic in [7] outperforms the two-stage algorithm, there is still room for improvement. In [7], it is a non-cooperative routing scheme where the receiver could only receive data from one sender. In [7], when the receiver received the data from multiple senders, the receiver could only select the data from the sender with the best signal quality. As a matter of fact, the receiver could combine the received signal from all the senders to get better signal quality by using the Maximum Ratio Combining (MRC) [8] signal processing technique. With this MRC technique, the signal power could be linearly combined so that the received Signal-to-Noise Ratio (SNR) is the sum of the SNR contributed from all the senders [8]. To be more specific, by leveraging on the MRC scheme, the power from multiple transmitters could be linear combined at the receiver. It could be expected that the cooperative routing scheme with this MRC technique could be more power efficient than the non-cooperative routing scheme. Figure 2 shows an illustrative example.

In Figure 2, we assume that the data rate demands for all the destination nodes are all 2 Mbps, and the available frequency spectrum at each node is $500 \mathrm{kHz}$. Hence, the transmitter must use at least the 16-QAM modulation to satisfy the destinations' bandwidth demands. We also assume that the nodes covered within the solid circle (denoted as SNR-aware transmission radius) could get sufficient SNR to decode the 16-QAM data, and the nodes covered within the dashed circle (denoted as transmission radius) but outside the solid circle could only get half of the required SNR.

In Figure 2a, every node is within node A's transmission coverage, so it is a feasible solution from the MPB's point of view. However, we could not satisfy the SNR (a) $\oplus$ source node

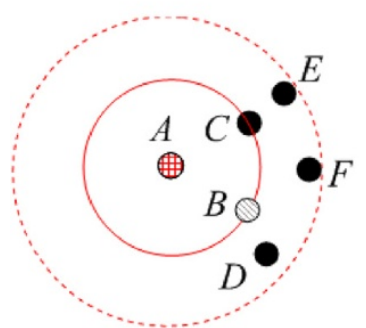

SNR requirement failed (b)

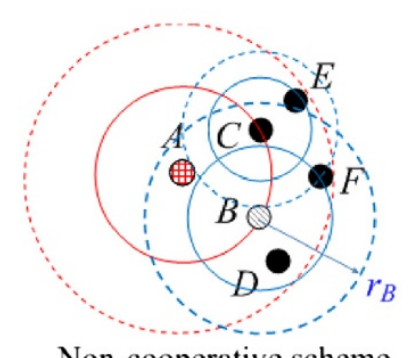

Non-cooperative scheme (c) relay nodes

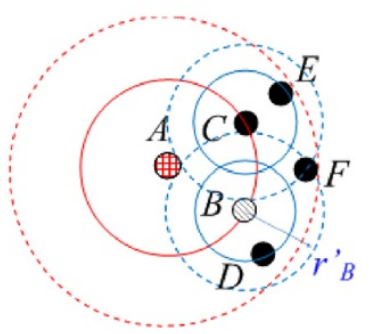

Cooperative scheme

Figure 2 SNR-aware power-efficient cooperative multicast routing. 
(bandwidth) requirement for nodes $D, E$, and $F$ if node $A$ transmits its signal with 16-QAM modulation scheme. Nodes $D, E$, and $F$ could not successfully decode the information because of the poor SNR. In other words, nodes $D, E$, and $F$ are within the transmission radius of $A$ but not within the SNR-aware transmission radius of $A$; therefore, it fails to meet the bandwidth QoS at nodes $D, E$, and $F$.

One possible way to satisfy the bandwidth requirement for all the nodes is shown in Figure $2 \mathrm{~b}$. In Figure 2b, nodes $B$ and $C$ turn on their power. Nodes $D$ and $F$ are within the SNR-aware transmission radius of node $B$, and node $E$ is within the SNR-aware transmission radius of node $C$. Then the SNR and bandwidth requirement for all the destination nodes could be satisfied. By carefully examining Figure $2 \mathrm{~b}$, we find that there is still room to further reduce the power consumption if the cooperative routing scheme is adopted. To be more specific, in Figure $2 \mathrm{~b}$, node $C$ contributes half of the required SNR to node $F$. If node $F$ could get the other half of the required SNR from node $B$, then the SNR requirement could be satisfied. To put it in a different way, by shrinking the transmission radius of node $B$ from $r_{B}$ to $r_{B}^{\prime}$ as shown in Figure 2c, node $F$ is within node $B$ 's transmission radius to get half of the required SNR. By adopting the MRC signal processing technique, the SNR at node $F$ is equal to the sum of the SNR contributed from nodes $B$ and $C$. Hence, the SNR at node $F$ could meet the minimum SNR requirement to successfully decode the received data by using the 16-QAM modulation scheme. In addition, node $D$ is still within node B's SNR-aware transmission radius. Then all the SNR requirements are all satisfied for the destination nodes. This example shows that cooperative routing scheme is more power efficient than the noncooperative routing scheme. To facilitate the cooperative routing scheme, a new many-to-one communication (e. g., from nodes $B$ and $C$ to $F$ ), denote as convergecasting, should carefully be designed.

In order to realize the collaborative communication as shown in Figure 2c, several issues needed to be addressed at the same time.

(1)Modulation scheme: Modulation scheme should carefully be selected by the transmitter to facilitate two important criteria. The first is to increase the data throughput at the receiver. The data throughput is determined by the frequency spectrum and the modulation scheme. Due to the limited frequency spectrum in wireless networks, adopting sophisticated modulation scheme is a good way to increase the data throughput at the receivers. The second is to make sure the receivers would successfully decode the information. Sophisticated modulation scheme requires better signal quality than ordinary modulation scheme. Hence, the transmitter that uses the sophisticated modulation scheme could cover fewer receivers than the transmitter that uses the ordinary modulation scheme under the same transmission radius. To summarize, there is a tradeoff between SNR-aware coverage and data throughput in selecting the modulation scheme.

(2)SNR-aware power control: From the power consumption point of view, the transmission power should be as minimal as possible to minimize the total power consumption. From the signal quality point-of-view, the transmission power should be as large as possible for the receiver to successfully decode the information. Hence, the power control strategy should carefully be designed to be SNRaware and at the same time to minimize the transmission power.

(3) WMA-enabled power efficient routing: Taking advantage of the WMA, the transmission power could significantly be reduced as compared to the wired line communication. Routing protocol in the wireless network should incorporate the WMA for power saving.

(4) Convergecasting with signal quality aggregation: By adopting the MRC signal processing technique, the SNR at the receiver is the sum of the contributed SNR from all the transmitters. This raises a new routing protocol (i.e., convergecasting) to facilitate the MRC technique for meeting the SNR requirement at the destination nodes. Unlike traditional one-to-many multicasting communication, convergecasting is a many-to-one communication strategy that requires totally new design philosophy and idea to be power aware and SNR aware simultaneously.

It is noted that the node with capability of selecting the modulation scheme (which is known as adaptive modulation) requires complicated hardware design. In other words, node with adaptive modulation capability is more expensive than the node without adaptive modulation. Besides expensive hardware cost, the additional adaptive modulation circuit incurs larger node processing power. Hence, it is not easy to ask every node in the wireless network to incorporate this adaptive modulation capability. In this article, we will assume that the modulation scheme is fixed for the nodes.

MPBBA problem via cooperative communication is a challenging cross-layer (layer $1+$ layer 3 ) design problem that includes SNR-aware transmission and power control in the physical layer and minimum power multicast and convergecast routing in the network layer. This 
article is a pioneer in addressing the MPBBA problem via cooperative communication by proposing integrated cross-layer heuristics that intelligently perform the SNRaware minimum power multicast and convergecast routing in wireless ad hoc network to meet the SNR requirements of the destination nodes. In the sequel, we summarize the related works on MPB, MPBBA, and cooperative routing problems.

Several related works address the MPB problem. In [1], three energy-efficient heuristic algorithms are proposed. They are the shortest path-based algorithm, the minimum spanning tree algorithm, and the broadcast/ multicast incremental power (MIP) algorithm. MIP algorithm exploits the WMA in wireless network via minimum incremental transmission power to cover unvisited node. According to the simulation results, shortest path algorithm can achieve excellent performance for small networks and the MIP algorithm works well for large networks. In [3], the performances of the above three algorithms are analytically evaluated. To get optimal solution, three integer linear programming models are proposed in [9]. However, no numerical results are reported to justify the applicability. By using CPLEX optimization solver to the optimization models in [9], we find that optimal solution can only be obtained for small network (less than 30 nodes) in days of computation. The MIP3S algorithm is proposed in [4]. By expanding the transmission power to cover a few more nodes (denote as set $\Phi$ ), potential power saving is possible by reducing the transmission radius of other nodes that previously cover the nodes in $\Phi$. It is shown that MIP3S performs better than MIP. However, the computational complexity of MIP3S is $O\left(|N|^{4}\right)$. It is higher as compared to $O\left(|N|^{3}\right)$ in MIP.

Recently, some works based on optimization technique to solve the MPB problem have been proposed. Yuan et al. [10] present a novel MILP model that leads to a sharp lower bound of the optimum via Lagrangian relaxation. In the same article, they also propose a heuristic algorithm named Successive Power Adjustment (SPA). The algorithm combines enhanced version of Sweep and Shrink algorithms to achieve a feasible upper bound solution. The computational complexity of SPA algorithm is $O\left(|N|^{3}\right)$. Another optimization-based approach for MPB problem is proposed in [5]. By leveraging on the information from the Lagrangian multiplier, we could construct more power-efficient routing paths. Numerical results demonstrate that the proposed approach in [5] outperforms the MIP and MIP3S for broadcast, multicast, and unicast communications. The above optimization-based approaches are designed for omnidirection antenna scenario. Algorithms for networks using directional antenna can be found in [11,12]. Due to many active research works proposed for resolving the MPB problem, for other kinds of approaches, please refer to the survey article for details [13]. As discussed in Figure 2, these MPB algorithms are not applicable to the MPBBA problem because the bandwidth requirement and signal quality are not considered.

In [7], to the best of the author's knowledge, this article is the first one to address the MPBBA problem. In [7], we tackle the MPBBA problem by using the noncooperative routing scheme where the receiver could only receive data from one sender. Hence, when the receiver received the data from multiple senders, the receiver could only select the data from the sender with the best signal quality. As discussed in Section "Introduction", by adopting the MRC scheme, the signal quality could linearly be combined at the receiver from multiple transmitters so that it is easier to satisfy the SNR requirement of the receiver. It could be expected that the cooperative routing scheme with this MRC technique could be more power efficient than the noncooperative routing scheme. In the computational experiments, it is also shown that the proposed cooperative routing algorithm, bandwidth-aware cooperative radius adjustment (BACRA), is more power efficient than the non-cooperative routing scheme as proposed in [7].

The study of Khandani et al. [14] is the first one that studies how energy saving is possible by using the cooperative transmission via WMA property in wireless networks. They devise the power cost function for the cooperative link and prove that the cooperative transmission could be more energy efficient than noncooperative point-to-point communication. They devise the CAN heuristic for this problem. The idea of CAN is to calculate the non-cooperative shortest path and then try to reduce the total transmission power via cooperation along this shortest path. The authors of [15] prove that the minimum energy cooperative path routing is an NP-complete problem and devise the Cooperative Shortest Path (CSP) heuristic to tackle this problem. The CSP is basically a Dijkstra shortest path algorithm with different link arc weight settings. The link arc weight is defined as the cooperative link cost as defined in [14]. They show that CSP has better performance than the CAN heuristic.

Unlike in $[14,15]$ that only assume single flow in the cooperative routing problem, in [16], the authors consider the multiple flows from multiple sources to multiple destinations. There would be MAC layer contention problem when neighboring flows use the same channel. They devise the heuristic to address this crosslayer (network layer + MAC layer) cooperative communication problem and conclude that the proposed algorithm is better than the single flow algorithm (e.g., CAN, CSP) in terms of network throughput.

As indicated in the previous paragraphs, all the existing literatures on cooperative routing do not address the 
signal quality or the bandwidth requirement. To the best of the author's knowledge, this article is the first one to address the MPBBA problem by using the cooperative routing scheme. In this article, for the first time, a cooperative routing heuristic algorithm, BACRA, to obtain the minimum power multicast routing in large wireless networks that meets the bandwidth QoS requirement is proposed. BACRA is proven to be optimal in terms of the SNR to power ratio and is superior to the other heuristics under all the tested cases in the computational experiments.

The remainder of this article is organized as follows. In the next section, the cooperative routing model is first studied to give the MPBBA problem formulation and then the BACRA heuristics that is based on maximizing the SNR to power ratio is proposed to minimize the energy consumption with the SNR requirements. In Section "Numerical results", the numerical results for different modulation schemes (SNR requirements) are tested under varieties of large random networks. Finally, a conclusion remark and future works are given.

\section{Cooperative routing model for MPBBA Cooperative routing model}

In cooperative routing path, there are three different kinds of links, namely point-to-point link, point-tomultipoint link, and multipoint-to-point link. Figure 3 shows an illustrative example of cooperative communication. At point-to-point link, there is only one transmitting node and receiving node (e.g., link $A \rightarrow B$ ). At point-to-multipoint link, there is one transmitting node and multiple receiving nodes (e.g., links $B \rightarrow C$ and $B \rightarrow D)$. Because of WMA property, the power consumption at node $B$ is $\max \left\{\prod\left(d_{B C}\right), \prod\left(d_{B D}\right)\right\}$, where $\prod\left(d_{B C}\right)$ is defined as the transmission power for node $B$ with transmission radius $d_{B C}$. At multipoint-to-point link, multiple transmitting nodes cooperatively transmit data to one receiving node (e.g., links $C \rightarrow E$ and $D \rightarrow E$ ). Note that since the cooperating routing path (e.g., Figure 3 ) is neither ordinary single path nor ordinary multicast tree, traditional path model or tree model is

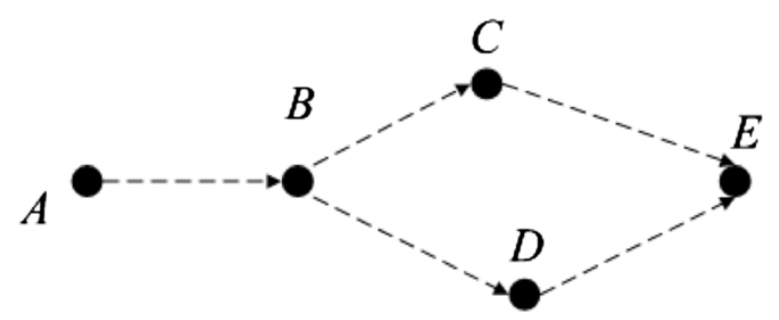

Figure 3 Cooperative routing path example. not able to characterize cooperative routing path. Then this raises an interesting question to be answered, what is the total cost for multipoint-to-point communication by using cooperative communication.

A new model needs to be devised to characterize the cooperative routing path especially for multipoint-topoint cooperative communication. Let $\operatorname{LC}(S, T)$ denote the total cost for nodes in $S=\left\{s_{1}, s_{2}, \ldots, s_{n}\right\}$ cooperatively transmit data to node in $T=\left\{t_{1}\right\}$. Khandani et al. [14] derive the minimum total link cost $\mathrm{LC}(S, T)$ to make sure the receiver could correctly decode the information only when the SNR at the receiver is above a minimum threshold $\mathrm{SNR}_{\min }$. In point-to-point communication (i.e., $S=\left\{s_{1}\right\}$ ), the link cost is

$$
\Pi\left(s_{1}\right)=\mathrm{LC}\left(s_{1}, t_{1}\right)=\left(\mathrm{SNR}_{\min }\right) \times P_{\eta} \times d_{1}^{\alpha},
$$

where $P_{\eta}$ denotes the noise power, $d_{1}$ is the distance between $s_{1}$ and $t_{1}$, and $\alpha$ denotes the power attenuation factor which is usually between 2 and 4 . Because it is a pointto-point communication, the link cost is equal to the required power at node $s_{1}$ (i.e., $\left.\Pi\left(s_{1}\right)\right)$. Given a modulation scheme, the minimum SNR to successfully decode the information is fixed (i.e., $\mathrm{SNR}_{\min }$ is fixed). In addition, $P_{\eta}$ is also constant. Then link cost $\Pi\left(s_{1}\right)$ is a function of distance $d_{1}$.

In the multipoint-to-point link, the minimum cooperative link cost $\mathrm{LC}(S, T)$ from nodes in $S=\left\{s_{1}, s_{2}, \ldots, s_{n}\right\}$ cooperatively transmit data to node $t_{1}$ is

$$
\begin{aligned}
\mathrm{LC}\left(S, t_{1}\right) & =\frac{1}{\sum_{i=1}^{n} \frac{1}{\left(\mathrm{SNR}_{\min }\right) \times P_{\eta} \times d_{i}^{\alpha}}}=\frac{1}{\sum_{i=1}^{n} \frac{1}{L C\left(s_{i}, t_{1}\right)}} \\
\text { If } \operatorname{LC}\left(s_{1}, t_{1}\right) & =\mathrm{LC}\left(s_{2}, t_{1}\right)=\cdots=\mathrm{LC}\left(s_{n}, t_{1}\right) \text {, we have } \\
\operatorname{LC}\left(S, t_{1}\right) & =\frac{L C\left(s_{1}, t_{1}\right)}{n}
\end{aligned}
$$

Based on Equation (3), we only need $(1 / n)$ of the original total transmission power to achieve the same SNR at node $t_{1}$ via the cooperative communication. In addition, the required power at each node $s_{i}$ is

$$
\Pi\left(s_{i}\right)=\frac{\frac{1}{L C\left(s_{i}, t_{1}\right)}}{\sum_{i=1}^{n} \frac{1}{L C\left(s_{i}, t_{1}\right)}} \times \frac{L C\left(s_{1}, t_{1}\right)}{n}=\frac{L C\left(s_{1}, t_{1}\right)}{n^{2}}
$$

Hence, each node only need $\left(1 / n^{2}\right)$ of the original transmission power to achieve the same SNR at node $t_{1}$ via the cooperative communication from $n$ transmitters. From Equations (3) and (4), we can conclude that cooperative communication is more energy efficient than non-cooperative communication not only from total transmission power, but also from individual transmission power at the transmitter. Hence, cooperative communication could not only reduce transmission power, but also implicitly prolong the system lifetime of the wireless networks. Based on these observations, 
cooperative communication should be encouraged as much as possible. This kind of energy saving is because of the WMA property in wireless networks.

Note that in the above power cost model for cooperative communication, the transmitters use the same modulation scheme to transmit the data to the receiver. This assumption is valid for the MRC scheme because the SNR could only be the sum of the SNR contributed from all the transmitters when they use the same modulation scheme.

Even though this model concludes that cooperative communication is more energy efficient than traditional non-cooperative communication, it only considers onehop communication. Hence, power control and multihop routing issues are not addressed to identify the minimum power routing path from the source to destination nodes. In the sequel, these two important issues to tackle this MPBBA problem by using the cross-layered cooperative routing scheme will be studied.

\section{MPBBA model}

The MPBBA problem via the cooperative scheme could formally be stated as: identifying the cooperative routing strategies to minimize total energy consumption subject to the bandwidth requirements of the destination nodes. The multicasting case is considered here; that is, there is one source node and multiple destination nodes with the same bandwidth demands.

Nodes without the adaptive modulation capabilities could significantly simplify the hardware complexity. This will save the hardware cost and node processing power. When the modulation scheme for every node in the network is fixed, then the minimum SNR to successfully decode the received data is also determined to meet the bandwidth requirement. In other words, when the modulation scheme is fixed, the MPBBA problem becomes to minimize total energy consumption subject to the SNR requirements of the destination nodes. This is a valid assumption because energy saving is the most important factor in wireless network. Subsequently, the MPBBA problem without the adaptive communication capability (i.e., modulation scheme is fixed for all nodes) will be focussed on.

Before introducing the MPBBA with SNR requirements and our proposed heuristics, the notations used in the heuristics are first defined.

Input values:

$N \quad$ the set of nodes

$G \quad$ the set of destination nodes

$R_{n} \quad$ the set of candidate transmission radius for node $n$

$\rho \quad$ the minimum SNR to successfully decode the received data
$\Psi\left(r_{n}, d_{n m}\right)$ the received SNR at node $m$, when node $n$ with transmission radius $r_{n}$ transmits data to node $m$ that is distance $d_{n m}$ away from node $n$

$\Pi\left(r_{n}\right) \quad$ transmission power for node $n$ with transmission radius $r_{n}$

$T \quad$ be the set of the network nodes; (i.e., $T=N$ )

Decision variables:

$T_{a} \quad$ be the set of the nodes that the SNR requirements are satisfied

$T_{b} \quad$ be the set of the nodes included in set $T$ but not included in set $T_{a}$ (i.e., $T_{b}=T-T_{a}$ )

$r_{n}^{B} \quad$ transmission radius assignment at node $n$ before transmission radius adjustment

$r_{n}^{A} \quad$ transmission radius assignment at node $n$ after transmission radius adjustment

$S_{m}^{B} \quad$ the aggregate SNR at node $m$ before any node $n$ in set $T_{a}$ increasing its transmission radius

$S_{m}^{A} \quad$ the aggregate SNR at node $m$ after any node $n$ in set $T_{a}$ increasing its transmission radius

The MPBBA problem with SNR requirements could be formulated as Problem $(P)$.

Problem $(P)$ :

$$
Z_{P}=\min \sum_{n \in N} \Pi\left(r_{n}\right)
$$

Subject to:

$$
\begin{array}{ll}
\rho \leq \sum_{n \in N, n \neq m} \Psi\left(r_{n}, d_{n m}\right) & \forall m \in G \\
r_{n} \in R_{n} & \forall n \in N .
\end{array}
$$

The objective function is to minimize the total transmission power. Equation (6) enforces that the SNR requirements should be satisfied for all the destination nodes. The cooperative communication is also facilitated in Equation (6) where the SNR for any destination node $m$ is contributed from the other nodes. Problem $(P)$ is an NP-complete problem because minimum energy cooperative routing problem is proven to be NP-complete problem [15] and minimum energy cooperative routing problem is a special case of problem $(P)$ when the SNR requirement is relaxed. A heuristic algorithm to tackle Problem $(P)$ in the following is proposed.

\section{Methods: BACRA algorithm}

The proposed algorithm is denoted as the BACRA algorithm. The basic idea of BACRA algorithm is to choose the node to increase the transmission radius that has the maximum increased SNR of the other nodes. Hence, the node in set $T_{a}$ that contributes the largest 
ratio of the increased SNR in set $T_{b}$ to the increased transmission power is selected to expand its transmission radius. This procedure is repeated until the SNR requirements are satisfied for all of the destination nodes, i.e., $G \subseteq T_{a}$.

In the beginning of the BACRA algorithm, we set $T$ to be all the networks nodes (i.e., $T=N$ ). The set $T_{a}$ includes all the nodes that receive SNR above the minimum threshold $\rho$. Because of $T_{b}=T-T_{a}$, all the network nodes not in set $T_{a}$ are included in set $T_{b}$, and the received SNR of these nodes in $T_{b}$ is below the minimum threshold $\rho$. Hence, the nodes in set $T_{a}$ could successfully decode the received data and transmit the data to the nodes in set $T_{b}$. When the node in set $T_{a}$ expands its transmission radius, the received SNR for some nodes in set $T_{b}$ might be increased so that the received SNR is above the minimum threshold $\rho$. These nodes in set $T_{b}$ meeting the SNR requirements will be included into the set $T_{a}$. This process will be iterated until all the destination nodes are included in set $T_{a}$. Then an important question to be asked is: which node in set $T_{a}$ should be selected to expand its transmission radius?

In the BACRA algorithm, we select the node $\alpha$ in set $T_{a}$ that can increase the largest ratio of the increased SNR in set $T_{b}$ to the increased transmission power. In other words,

$$
\alpha=\arg \left\{\operatorname{Max}_{n \in T_{a}}\left(\frac{\sum_{m \in T_{b}} \operatorname{Min}\left(S_{m}^{A}-S_{m}^{B}, \rho-S_{m}^{B}\right)}{\Pi\left(r_{n}^{A}\right)-\Pi\left(r_{n}^{B}\right)}\right)\right\}
$$

In the numerator of Equation (8), it is equal to the summation of the increased SNR in set $T_{b}$. In bandwidth-aware transmission, when the modulation scheme is fixed, the data rate is fixed when the received SNR is over the SNR threshold $\rho$. In other words, when the SNR requirement is satisfied, increasing the SNR will not increase the data rate. Hence, the received SNR for each node should be upper bounded by $\rho$. Therefore, the increased SNR is the minimum of these two values (i.e., $\left.\operatorname{Min}\left(S_{m}^{A}-S_{m}^{B}, \rho-S_{m}^{B}\right)\right)$. The denominator of Equation (9) is the increased transmission power. This increased SNR to increased power ratio (SNR/P) is basically the larger the better, because it indicates the efficiency of increased transmission power to the increased SNR. By selecting the node in set $T_{a}$ with the largest $\mathrm{SNR} / \mathrm{P}$ ratio to expand its transmission radius first would implicitly achieve the objective of minimum transmission power as well as to meet the bandwidth/SNR requirements of the destination nodes.

There are three properties in Equation (8).

\section{Meeting the SNR requirements before transmitting:} The transmitter is selected from the set $T_{a}$ so as to ensure that the transmitter has enough SNR to successfully decode the information before transmitting.

2. Power control with respect to the SNR/P: The numerator of Equation (8) is the total increased SNR in set $T_{b}$. The denominator of Equation (8) is the increased transmission power. Then Equation (8) is to select the node in set $T_{a}$ to expand its transmission radius so that it contributes the largest ratio SNR/P. In other words, the selection of transmission node and its transmission power is based on the performance measure SNR/P.

3. No loopback: It ensures after selecting a node in set $T_{a}$ to expand its transmission radius, it is not possible to reduce the transmission radius of the other node in set $T_{a}$ to further increase the SNR/P. In other words, selecting the node in set $T_{a}$ to expand its transmission radius at the later stage will not affect the transmission power assignment for the selected node in set $T_{a}$ at the earlier stage. This is called the no loopback property. This no loopback property is important to make sure that the BACRA algorithm does not have infinite looping problem. The no loopback property is proven in the Appendix.

Based on these three properties, we claim that the BACRA algorithm is optimal in terms of SNR/P performance measure. We do not claim that the BACRA algorithm is optimal to the MPBBA problem because adaptive modulation scheme is not considered in Problem $(P)$. However, because SNR/P indicates the contributed SNR by additionally increased transmission power, which addresses the signal quality and transmission power at the same time, we will show in the computational experiments that BACRA algorithm is superior to the other heuristics at the MPBBA problem.

Next we perform the complexity analysis for the BACRA algorithm. First, we study the time complexity. It is clear that the most time-consuming part of the BACRA algorithm is the "While" loop. So, we need to know what is the total number of iterations for the "While" loop. In the worst case, the destination node will be the last one to be included to the set $T_{a}$. In this case, all the network nodes are examined. In other words, the "While ( $G \not \subset \mathrm{T}_{\mathrm{a}}$ and $\alpha \neq 0)$ " has to loop for $(|N|-1)$ times. Next, we observed that there are two steps in the "While" loop. In step 1, there are two layers of "For" loop. In the worst case, there are $(|N| / 2)$ number of nodes in set $T_{a}$ and $\left({ }^{|N|} / 2\right)$ number of nodes in set $T_{b}$. In the worst case, the total number of iterations would be $\left(|N|^{2} /{ }_{4}\right)$. Then the computational complexity for step 1 is $O\left(|N|^{2}\right)$. In step 2, there is only one layer of "For" loop. In the worst case, 
Table 1 Complexity analysis of routing information dissemination and collection in BACRA

\begin{tabular}{|c|c|c|c|}
\hline & Convergence time & Memory overhead & Control overhead \\
\hline$\overline{O S L R}[17]$ & $O(D \cdot I)$ & $O\left(|N|^{2}\right)$ & $O\left(|N|^{2}\right)$ \\
\hline
\end{tabular}

$\overline{|N|}$ number of nodes in the network, $I$ average update interval, and $D$ diameter of the network.

there are $(|N|-1)$ number of nodes in set $T_{b}$. The total number of iterations would be $(|N|-1)$ for step 2 . Then the computational complexity for step 2 is $O(|N|)$. The "While $G \not \subset \mathrm{T}_{\mathrm{a}}$ and $\alpha \neq 0$ )" has to loop for $(|N|-1)$ times in the worst case and the computational complexity of step 1 is $O\left(|N|^{2}\right)$. The computational complexity for the BACRA algorithm is $O\left(|N|^{3}\right)$.

Second, we study the message complexity (communication cost) of the BACRA algorithm. Two sets of information that requires message communication between the nodes in our BACRA algorithm are the transmission radius configuration for each node $n$ (i.e., $r_{n}$ and $\Pi\left(r_{n}\right)$ ) and the received SNR for each node $m$ (i.e., $\Psi\left(r_{n}, d_{n m}\right)$ ). These two sets of information, transmission radius configuration and the received SNR information, varies over time and changes from time-to-time in the BACRA algorithm. These two sets of information should be broadcasted periodically to the other nodes. Instead of using the flooding scheme for broadcasting, we adopt the OSLR routing protocol [17] to disseminate the control messages. OSLR protocol proposes a multipoint relaying strategy to minimize the size of the control message and the number of rebroadcasting nodes. By utilizing these two sets of control messages, the signal quality of the wireless network could be acquired timely to determine the available bandwidth. According to the OSLR protocol, the message complexity analysis is summarized in Table 1.

\section{BACRA algorithm}

Begin

Input: Network topology, SNR requirements for each destination node;

Output: Routing assignment and transmission radius for each node;

Let set $T$ be the set of the networks nodes; //T=N

$T_{a}=$ \{source node $\}$ and $T_{b}=T-$ source node $\} ;$

For $(n \in T) / /$ initialize $r_{n}^{A}, r_{n}^{B}, S_{m}^{A}$ and $S_{m}^{B}$

Begin

$$
r_{n}^{B}=0 ; S_{m}^{A}=0 ; S_{m}^{B}=0 ;
$$

Let $r_{n}^{A}$ be the smallest transmission radius in set $R_{n}$;

End//For $\alpha=1$

While ( $G \not \subset \mathrm{T}_{\mathrm{a}}$ and $\left.\alpha \neq 0\right)$

Begin

$k=0 ; \alpha=0$;

For $\left(n \in T_{a}\right) / /$ step 1 : identify the node that contributes maximum increased SNR to the nodes in set $T_{b}$

Begin

For $\left(m \in T_{b}\right)$

Begin

$S_{m}^{A}=\Psi\left(r_{n}^{A}, d_{n m}\right)+S_{m}^{B}$

End; //For $m$

$$
\begin{aligned}
& \text { If }\left(k<\frac{\sum_{m \in T_{b}} \operatorname{Min}\left(S_{m}^{A}-S_{m}^{B}, \rho-S_{m}^{B}\right)}{\Pi\left(r_{n}^{A}\right)-\Pi\left(r_{n}^{B}\right)}\right) \\
& k=\frac{\sum_{m \in T_{b}} \operatorname{Min}\left(S_{m}^{A}-S_{m}^{B}, \rho-S_{m}^{B}\right)}{\Pi\left(r_{n}^{A}\right)-\Pi\left(r_{n}^{B}\right)} ; \alpha=n ;
\end{aligned}
$$

End;//For $n$, end of step 1

If $(\alpha \neq 0) / /$ step 2: node $\alpha$ contributes maximum increased SNR, adjust $r_{\alpha}^{B}, r_{\alpha}^{A}, S_{m}^{B}, T_{B}, T_{A}$

Begin

$r_{\alpha}^{B}=r_{\alpha}^{A}$;//adjust the transmission radius of node $\alpha$

$r_{\alpha}^{A}$ moves on to next larger transmission radius in set

$\mathrm{R}_{\mathrm{n}}$; //adjust the transmission radius of node $\alpha$

For $\left(m \in T_{b}\right) / /$ adjust $S_{m}^{B}, T_{b}$ and $T_{a}$

Begin

If $\left(S_{m}^{B}+\Psi\left(r_{\alpha}^{B}, d_{\alpha m}\right) \geq \rho\right) / /$ node $m$ 's SNR is satisfied, then include node $m$ in the set $T_{A}$

$S_{m}^{B}=\rho ; T_{b}=T_{b}-\{m\} ; T_{a}=T_{a} \cup\{m\} ;$

Else

$S_{m}^{B}=S_{m}^{B}+\Psi\left(r_{\alpha}^{B}, d_{\alpha m}\right)$

End;//For $m$

End;//If $(\alpha \neq 0)$

Else $/ / \alpha=0$

Report "infeasible solution";

End//while

End 


\section{Numerical results}

We have carried out a performance study on the MPBBA problem by using the BACRA approach, and drawn comparisons with MPB-based heuristics (MIP, MLU, MLiMST in [1], and MIP3S in [4]) and noncooperative MPBBA heuristics (LGR in [7]) via experiments over a randomly generated network. These MPB-based heuristics have been modified to address the bandwidth QoS requirement for fair comparison because these heuristics do not address the bandwidth QoS requirement.

First, the routing and the transmission radius assignments are obtained by the original algorithm. Then if the bandwidth QoS could not be satisfied on any relay node or destination node in the routing path, the other nodes on the routing path that meet the bandwidth QoS will try to transmit the power to this node with bandwidth QoS. In the next phase, the transmission radius is adjusted for possible transmission power reduction. Hence, these revised MPB-based heuristics are two-phase algorithms where the first phase is to determine the routing path and the second phase is to adjust the transmission radius to satisfy the bandwidth QoS requirement. Because of this two-phase adjustment to address the bandwidth QoS, we call these algorithms as MIP2, MLU2, MLiMST2, and MIP3S2, respectively.

We illustrate the modification process with an example in Figure 4. In Figure 4a, the original routing assignment for these four traditional MPB algorithms is to send the data to $C$ and then from $C$ to $D$. Because $C$ is not within the SNR-aware transmission radius of $A$, it fails to meet the bandwidth QoS at node $C$. In this case, $D$ is unreachable because $C$ is not able to transmit the data to $D$ with bandwidth guarantee. In the first phase, we expand $B$ 's transmission radius since $B$ is within the SNR-aware transmission radius of $A$. Then $C$ could transmit the data to $D$. In the second phase, by shrinking the transmission radius of $A$ so that $B$ is at the edge of the SNR-aware transmission radius, it could be more energy efficient.
Table 2 SNR for modulation scheme in WiMAX $[18,19]$

\begin{tabular}{cccc}
\hline Modulation & Code rate & $\begin{array}{c}\text { Achievable data rate } \\
(\mathbf{2 0 ~} \mathbf{~ M H z})\end{array}$ & SNR (in dB) \\
\hline QPSK & $1 / 2 \mathrm{CTC}$ & $20 \mathrm{Mbps}$ & $8<\mathrm{SNR} \leq 9.4$ \\
QPSK & $3 / 4 \mathrm{CTC}$ & $30 \mathrm{Mbps}$ & $9.4<\mathrm{SNR} \leq 11.2$ \\
16 QAM & $1 / 2 \mathrm{CTC}$ & $40 \mathrm{Mbps}$ & $11.2<\mathrm{SNR} \leq 16.4$ \\
16 QAM & $3 / 4 \mathrm{CTC}$ & $60 \mathrm{Mbps}$ & $16.4<\mathrm{SNR} \leq 18.2$ \\
64 QAM & $1 / 2 \mathrm{CTC}$ & $60 \mathrm{Mbps}$ & $18.2<\mathrm{SNR} \leq 22.7$ \\
64 QAM & $3 / 4 \mathrm{CTC}$ & $90 \mathrm{Mbps}$ & $22.7<\mathrm{SNR}$ \\
\hline
\end{tabular}

The SNR (in dB) could be calculated as follows.

The network nodes are randomly placed in the $25 \times$ $25 \mathrm{~km}^{2}$ area. Transmission power $\Pi\left(r_{n}\right)$ is set to $r_{n}^{\alpha}$, where the signal power attenuation constant $\alpha=3$. The set of possible communication radius is a discrete set starting from zero with step size 0.5 to $3.0 \mathrm{~km}$. We borrow the SNR parameters from WiMAX $[18,19]$ for the supported modulation scheme at Table 2. Note that the proposed algorithm could be applicable to any wireless network (e.g., ad hoc network). The reason that we choose the SNR parameters from WiMAX is because there is a clear specification for the SNR ratio and the associated modulation scheme.

The SNR (in $\mathrm{dB}$ ) could be calculated as follows,

$$
\text { SNR }=P_{t}+G_{t}+G_{r}-P L-\text { Noise }
$$

where $P_{t}$ indicates the transmission power, $G_{t}$ indicates the antenna gain in the transmitter, $G_{r}$ indicates the antenna gain in the receiver, $P L$ indicates the path loss, and Noise indicates the thermal noise power.

In the following experiments, the path loss (in $\mathrm{dB}$ ) is based on the measurements in line-of-sight (LOS) transmission in WiMAX [20].

$$
P L=110.11+21.29 \times \log \left(d_{t r}\right)
$$

where $d_{t r}$ indicates the distance (in kilometers) between the transmitter $t$ and receiver $r$.

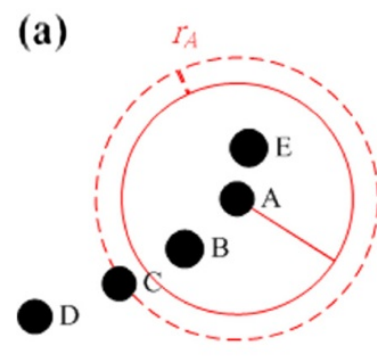

$D$ unreachable

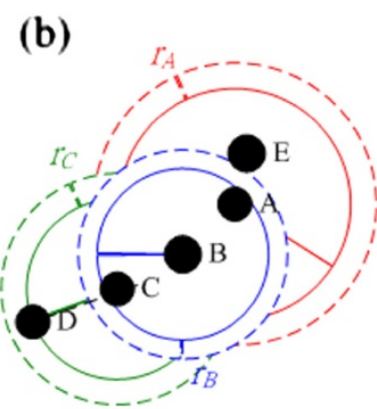

Phase 1: Expand B's radius (c)

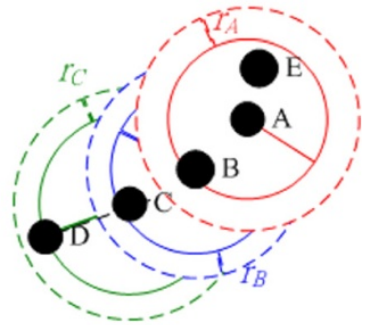

Phase 2: Shrink A's radius

Figure 4 Two-phase modification on MPB algorithms. 
The thermal noise power Noise in room temperature is

$$
\text { Noise }=-174 \mathrm{dBm}+10 \times \log (B)
$$

When bandwidth $(B)$ is $20 \mathrm{MHz}$, Noise is equal to $-101 \mathrm{dBm}$. Under the assumption of unity antenna gain, $G_{t}=G_{r}=0$, then we get

$$
\boldsymbol{S N R}=P_{t}-9-21.29 \times \log \left(d_{t r}\right)
$$

Then the variables in Problem $(P)$ of Section "Numerical results" become

$$
\begin{aligned}
& \Pi\left(r_{t}\right)=P_{t}=r_{t}^{\alpha} \\
& \Psi\left(r_{t}, d_{t r}\right)=\Pi\left(r_{t}\right)-9-21.29 \times \log \left(d_{t r}\right)
\end{aligned}
$$

In the following experiments, we assume that the available frequency spectrum is $20 \mathrm{MHz}$. Because of the fixed allocated frequency spectrum $(20 \mathrm{MHz})$, different modulation scheme requirements mean different bandwidth requirements. Then the achievable data rate with respect to different modulation scheme is shown in the third column of Table 2. For example, when the $\mathrm{SNR}=9 \mathrm{~dB}$, the modulation scheme is QPSK with $1 / 2$ CTC code rate according to Table 2 . Then we have

$$
20 \mathrm{MHz} \times 2 \mathrm{bps} / \mathrm{Hz} \times 1 / 2=20 \mathrm{Mbps}
$$

In the first set of experiments, the total number of nodes is 2,000. We study the power consumption with respect to the number of destination nodes in the multicast group for different modulation schemes. We have different bandwidth requirements from Figures 5, 6, and 7 , which is 20, 40, and $60 \mathrm{Mbps}$, respectively. From Figures 5, 6, and 7, there are three important observations.

\section{Larger transmission power in sophisticated} modulation scheme: In sophisticated modulation scheme, the SNR requirements are higher than the ordinary modulation scheme. According to the

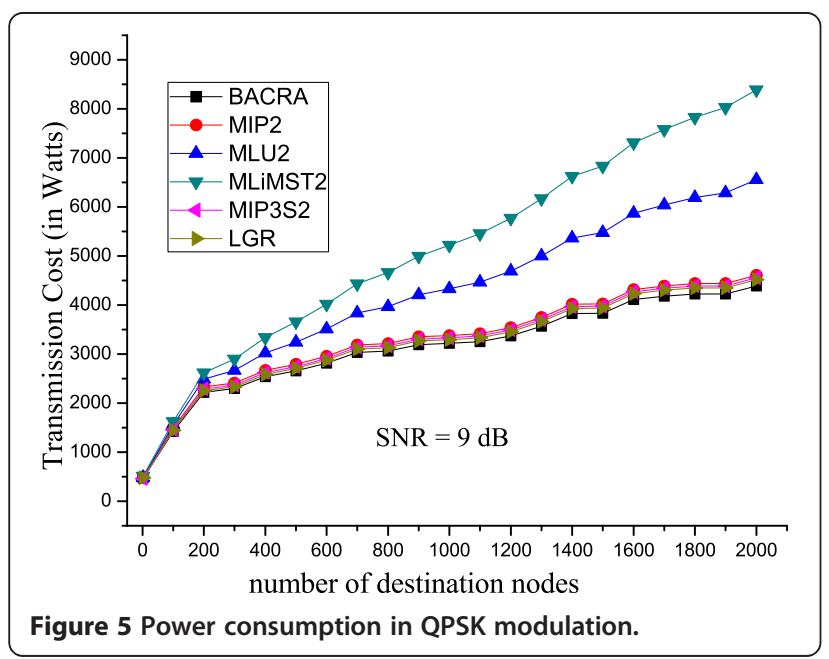

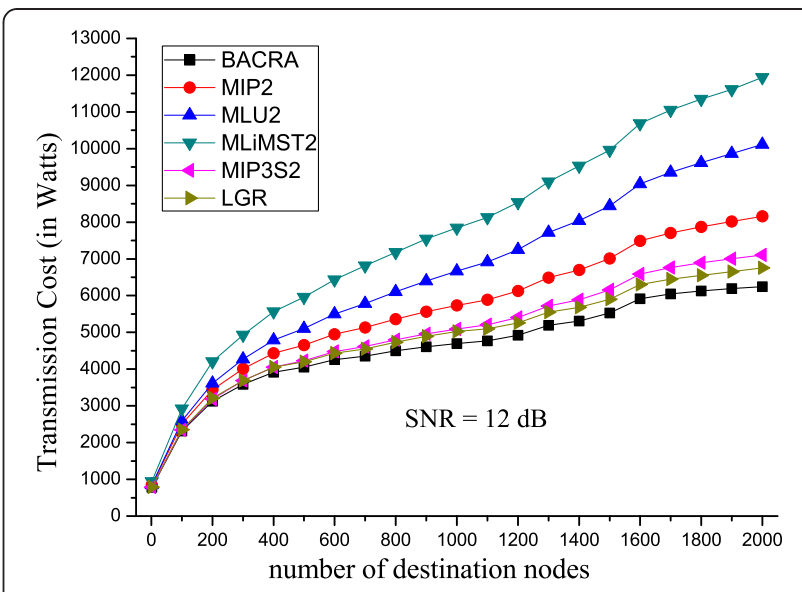

Figure 6 Power consumption 16 QAM modulation.

results in these three figures, it is observed that each algorithm will get larger total transmission power in more stringent SNR requirements.

2. Saturated power increasing at larger number of destination nodes: When in smaller number of destination nodes (e.g., 100 to 200 nodes), the transmission power is almost linearly increasing. When the number of destination nodes are close to the broadcasting case (e.g., 1,900 to 2,000 nodes), the transmission power will be saturated to an upper value especially in high SNR requirements (i.e., 64 QAM modulation). It is intuitive to have this result at larger number of destination nodes since most of the nodes in the network are transmitted at the maximum power so that the destination nodes could meet the SNR requirements.

3. Superior performance of BACRA to the other algorithms: From Figures 5, 6, and 7, we observe BACRA outperforms the other algorithms. We define the superior performance ratio (SR) to be the

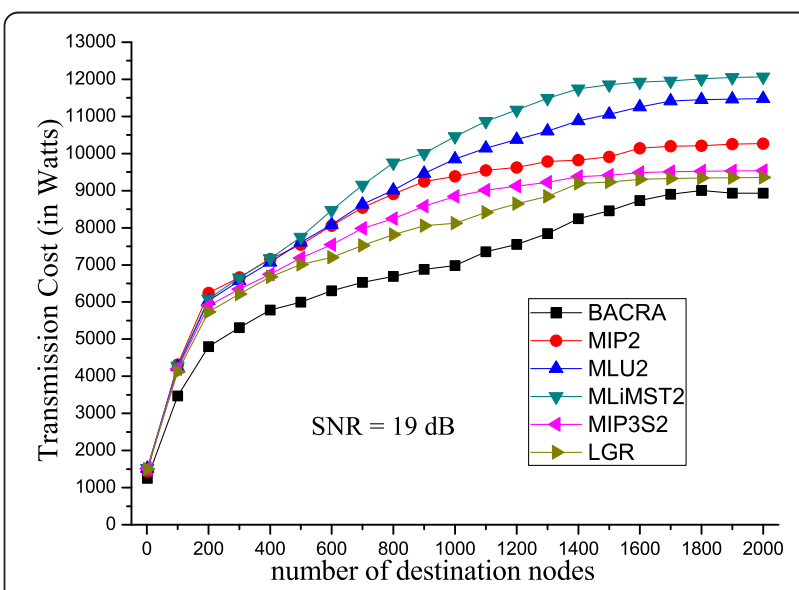

Figure 7 Power consumption in 64 QAM modulation. 
Table 3 SR

\begin{tabular}{cccc}
\hline SR of BACRA over & QPSK (\%) & 16 QAM (\%) & 64 QAM (\%) \\
\hline MLU2 & 49 & 62 & 41 \\
MLiMST2 & 91 & 91 & 49 \\
MIP2 & 5 & 31 & 34 \\
MIP3S2 & 4 & 14 & 27 \\
LGR & 3 & 8 & 20
\end{tabular}

performance metric for making comparison with the other five algorithms where $\mathrm{SR}=(\bar{A}-\bar{B}) / \bar{B}$ in percentage, where $\bar{B}$ and $\bar{A}$ are the mean transmission power of the BACRA algorithm and the other algorithms. We summarize SR at Table 3. From Table 3, we found that we have more significant power reduction (i.e., at least $20 \%$ improvement) at 64-QAM modulation scheme. This is because unlike the two-phase MPB heuristics and non-cooperative LGR, the BACRA algorithm considers the signal quality via the cooperative communication scheme in determining the routing path so that it is easier to identify the energy efficient path in stringent SNR requirements.

In the second set of experiments, we study the power consumption with respect to the network density for different modulation schemes. Intuitively, in the dense network (e.g., 2,700 nodes), the distance between the nodes is smaller so that it is easier to satisfy the SNR requirements. On the other hand, in the sparse network (e.g., 1,500 nodes), the distance between the nodes is larger so that more transmission power is needed to satisfy the SNR requirements. In this set of experiments, broadcasting case is considered where every node in the network is the destination node. Through this setting, we try to explore the impact of network

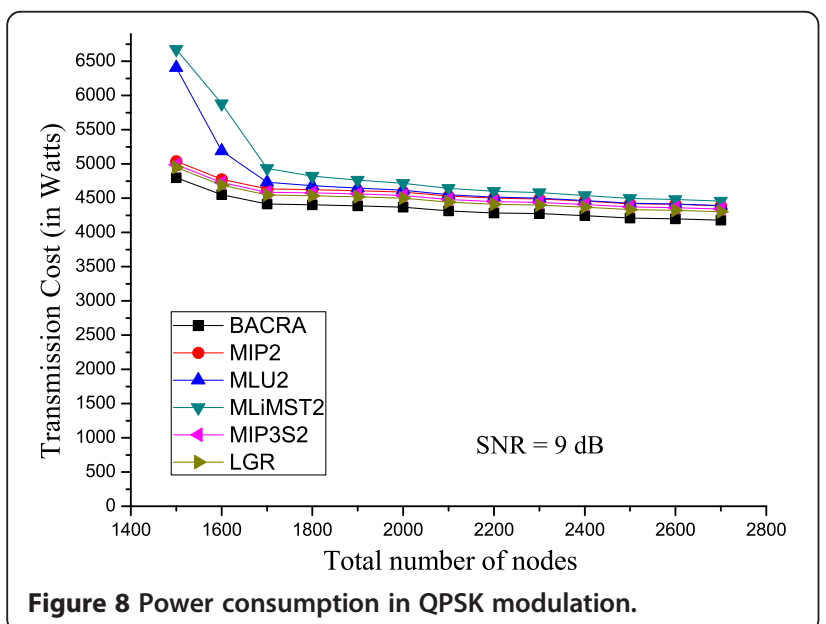

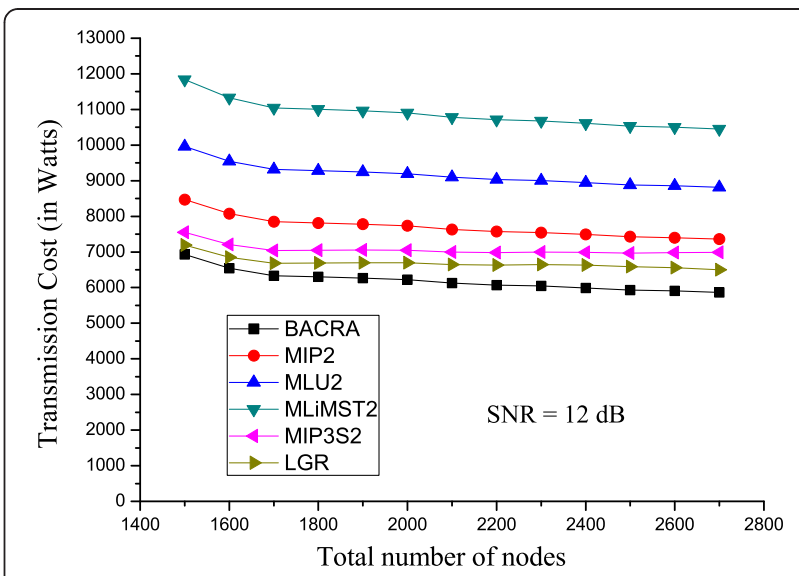

Figure 9 Power consumption in 16-QAM modulation.

density with respect to different SNR requirements in the broadcasting case.

As shown in Figures 8, 9, and 10, we have two important observations.

1. Larger transmission power in sparse networks than in dense networks: The computational results from Figures 8, 9, and 10 are intuitive that larger transmission power is needed in sparse network to meet the SNR requirements. Not only the transmission power is larger in the sparse network, but also it is not easy to identify feasible solutions when the SNR requirements are stringent. For example, at 64-QAM modulation $(\mathrm{SNR}=19 \mathrm{~dB})$, two-phase MPB heuristics (MIP2, MLU2, MLiMST, and MIP3S2) could not locate feasible solutions when the number of nodes is smaller than 1500 , 1700, 1800, and 1500 nodes, respectively.

2. Superior performance of BACRA over the other algorithms: We summarize the performance comparison of BACRA and the other algorithms at

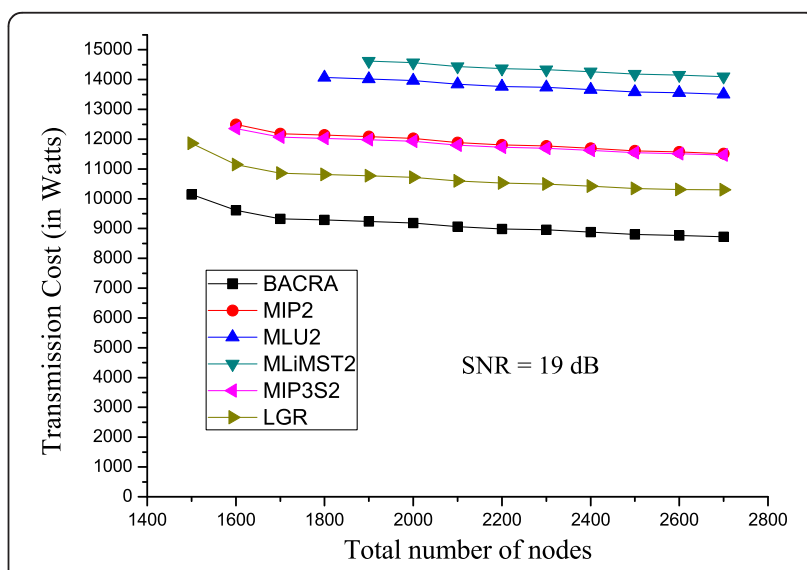

Figure 10 Power consumption in 64-QAM modulation. 
Table 4 SR with respect to network density

\begin{tabular}{cccc}
\hline SR of BACRA over & QPSK (\%) & 16 QAM (\%) & 64 QAM (\%) \\
\hline MLU2 & 33 & 50 & 55 \\
MLiMST2 & 39 & 78 & 62 \\
MIP2 & 5 & 25 & 32 \\
MIP3S2 & 4 & 19 & 31 \\
LGR & 3 & 11 & 18 \\
\hline
\end{tabular}

Table 4. It is observed that BACRA algorithm is superior to the other algorithms at all the tested cases, especially in 64-QAM modulation. Unlike the other MPB heuristics, the BACRA algorithm could still locate feasible solution at 1,500 nodes. Note that the SR value at the 64-QAM is calculated only in feasible solutions.

\section{Conclusions and future work}

Existing MPB algorithms without addressing the signal quality transmission are not applicable to the emerging wireless applications with bandwidth QoS requirements. In this article, for the first time, the novel SNR-aware and power-aware cooperative multicasting routing scheme, BACRA, is proposed to tackle this MPBBA problem. The basic idea of BACRA algorithm is to choose the node to expand its transmission radius that can increase the largest ratio of the increased SNR to the increased transmission power. Unlike the other heuristics that are two-phase algorithms and do not take advantage of cooperative communication, BACRA is an integrated cross-layer design algorithm that realize the advantage of cooperative communication via MRC scheme. Through optimizing the SNR/P performance measure, BACRA implicitly considers the SNR-aware power control and energy-aware cooperative routing simultaneously to meet the SNR requirements in energy-efficient ways. In addition, as compared to the two-phase MPB heuristics and non-cooperative LGR, the BACRA algorithm intelligently considers the signal quality via the cooperative communication scheme in determining relay nodes so that it is easier to identify the energy-efficient path in sparse network and stringent SNR requirements. According to the computational results, the proposed BACRA algorithm is superior to the other heuristics, especially in sparse network and stringent $\mathrm{SNR}$ requirements.

In the MPB and the MPBBA problems, they consider the path loss and attenuation in the signal propagation impairments. Besides path loss and attenuation, the signal transmission impairments in wireless network also include shadowing, multipath propagation, and interference. Shadowing occurs when there are obstacles between the transmitter and receiver so that there is no LOS transmission. Multipath propagation indicates that besides LOS transmission, there is non-LOS transmission that might incur inter-symbol interference. Interference indicates that some other signal on the same frequency band might interfere with the desired signal. Even though these three impairments play a non-negligible role in signal transmission, we only focus on the path loss and attenuation for simplifying the MPBBA model. This simplifying would enable us to grasp the whole picture more easily and understand the basic idea of the MPBBA problem as compared to the MPB problem. In the future, we will also consider other transmission impairments in the MPBBA problem so that it is more applicable in real wireless applications.

\section{Appendix}

Proof of Property 2 (no loopback property) in Section "Numerical results"

Prove by contradiction.

Given $n_{1}, n_{2} \in T_{a}, n_{1}$ is selected at the earlier stage and then $n_{2}$ is selected at the later stage with transmission radius $r_{n_{2}}^{A}$. Now assume that after $n_{2}$ is selected, we could decrease the transmission radius of $n_{1}$ for further increasing the SNR/P ratio. In other words,

$$
\begin{gathered}
\left(\frac{\sum_{m \in T_{b}} \operatorname{Min}\left(\overline{S_{m}^{A}}-S_{m}^{B}, \rho-S_{m}^{B}\right)}{\Pi\left(r_{n_{2}}^{A}\right)-\Pi\left(r_{n_{2}}^{B}\right)}\right. \\
\left.>\frac{\sum_{m \in T_{b}} \operatorname{Min}\left(S_{m}^{A}-S_{m}^{B}, \rho-S_{m}^{B}\right)}{\Pi\left(r_{n_{2}}^{A}\right)-\Pi\left(r_{n_{2}}^{B}\right)}\right)
\end{gathered}
$$

where $\overline{S_{m}^{A}}$ indicates the aggregate SNR at node $m$ after $n_{2}$ increases its transmission radius; $\overline{S_{m}^{A}}$ indicates the new aggregate SNR at node $m$ after $n_{2}$ increases its transmission radius and $n_{1}$ decreases its transmission radius.

By decreasing the transmission radius of the node $n_{1}$, the received aggregate SNR of the any node $m$ in set $T_{b}$ will either be the same or be decreased. In other words, $\overline{S_{m}^{A}} \leq S_{m}^{A}$. Then, Equation (15) will not hold. This violates the initial assumption that after $n_{2}$ is selected, we could decrease the transmission radius of $n_{1}$ for further increasing the SNR/P ratio.

This proves the no loopback property.

Competing interests

The authors declare that they have no competing interests.

\section{Acknowledgment}

This work was supported by the National Science Council, Taiwan, under grant number NSC 100-2622-H-128-002-CC3 and NSC 101-2410-H-128-005-MY2. 


\section{References}

1. J.E. Wieselthier, G.D. Nguyen, A. Ephremides, On the construction of energy-efficient broadcast and multicast trees in wireless networks, in Proc. IEEE. INFOCOM, 2000, Tel-Aviv, Israel (2000), pp. 585-594

2. W. Liang, Constructing minimum-energy broadcast trees in wireless ad hoc networks, in Proc. ACM. MobiHoc, 2002, Lausanne, Switzerland, 2002, pp. 112-122

3. P.-J. Wan, G. Călinescu, X.-Y. Li, O. Frieder, Minimum-energy broadcast routing in static ad hoc wireless networks, in Proc. IEEE. INFOCOM, 2001, Anchorage, USA (2001), pp. 1162-1171

4. P. Mavinkurve, H.Q. Ngo, H. Mensa, MIP3S: algorithms for power-conserving multicasting in wireless ad hoc networks, in Proc. IEEE. Int. Conf. Netw. (ICON), 2003, Sydney, Australia, 2003, pp. 143-148

5. H.H. Yen, S.S.W. Lee, F.G.H. Yap, A novel optimization-based approach for minimum power multicast in wireless networks. J. Commun. Netw. 13(1), 26-31 (2011)

6. Y. Xiao, J. Rosdahl, Throughput and delay limits of IEEE 802.11. IEEE Commun. Lett. 6(8), 355-357 (2002)

7. H.H. Yen, S.S.W. Lee, F.G.H. Yap, A novel optimization-based bandwidth-aware minimum power multicast routing algorithm in green wireless networks. J. Supercomput., (2012). doi:10.1007/s11227-011-0629-x

8. A. Goldsmith, Wireless Communications (Cambridge University Press, Cambridge, MA, 2005), pp. 214-216

9. A.K. Das, R.J. Marks, M. El-Sharkawi, P. Arabshahi, A Gray, Minimum power broadcast trees for wireless networks: integer programming formulations, in Proc. IEEE INFOCOM, 2003, San Francisco, USA (2003), pp. 1001-1010

10. D. Yuan, J. Bauer, D. Haugland, Minimum-energy broadcast and multicast in wireless networks: an integer programming approach and improved heuristic algorithms. Ad Hoc Netw 6, 696-717 (2008)

11. F. Dai, Q. Dai, J. Wu, Power efficient routing trees for ad hoc wireless networks using directional antenna. Ad Hoc Netw 3, 621-628 (2005)

12. S.G. Guo, O. Yang, Minimum-energy multicast in wireless ad hoc networks with adaptive antennas: MILP formulations and heuristic algorithms. IEEE Trans. Mob. Comput. 5, 333-346 (2006)

13. S.G. Guo, O. Yang, Energy-aware multicasting in wireless ad hoc networks: a survey and discussion. Comput. Commun. 30, 2129-2148 (2007)

14. A.E. Khandani, E. Modiano, J. Abounadi, L. Zheng, Cooperative routing in wireless networks, in Advances in Pervasive Computing and Network (Kluwer Academic Publishers, Boston, 2004)

15. F. Li, K. Wu, A. Lippman, Minimum energy cooperative path routing in all-wireless networks: NP-completeness and heuristic algorithm. J. Commun. Netw. 10(2), 1-9 (2008)

16. J. Zhang, Q. Zhang, Cooperative routing in static wireless networks, in Proc IEEE. INFOCOM, 2008, Phoenix, USA (2008), pp. 2369-2377

17. P. Jacquet, P. Muhlethaler, T. Clausen, A. Laouiti, A. Qayyum, L. Viennot, Optimized link state routing protocol for ad hoc networks, in Proc. of IEEE INMIC, 2001, Lahore, Pakistan (2001), pp. 62-68

18. WiMax Forum, WiMax Forum. http://www.wimaxforum.org

19. IEEE Std 802.16-2009, IEEE Standard for Local and metropolitan area networks - Part 16: Air 15 Interface for Broadband Wireless Access Systems.

20. P. Grønsund, P.E. Engelstad, T. Johnsen, T. Skeie, The physical performance and path loss in a fixed WiMAX deployment, in Proc. Int. Conf. Wireless. Commun. Mobile. Comput, 2007, Hawaii, USA (2007), pp. 439-444

Submit your manuscript to a SpringerOpen ${ }^{\odot}$ journal and benefit from:

- Convenient online submission

- Rigorous peer review

- Immediate publication on acceptance

- Open access: articles freely available online

- High visibility within the field

- Retaining the copyright to your article

Submit your next manuscript at $\gg$ springeropen.com 